\title{
Investigating the dosimetric effect of inter-fraction deformation in lung cancer stereotactic body radiotherapy (SBRT)
}

\author{
Jing Jia, Zhen Tian, Xuejun Gu, Hao Yan, Xun Jia and Steve Jiang \\ Department of Radiation Oncology, UT Southwestern Medical Center, Dallas, TX, USA.
}

Received March 19, 2014; Published Online April 08, 2014

[Presented at the Young Investigator's Symposium at the 2014 Annual Meeting of Southwest Chapter of American Association of Physicists in Medicine (AAPM) in San Antonio, Texas, USA]

\section{Conference Proceeding}

\begin{abstract}
Purpose: We studied on the negative dosimetric effect of inter-fraction deformation in lung stereotactic body radiotherapy (SBRT), in order to see whether there is a need for adaptive re-planning of lung SBRT cases.

Methods: Six lung cancer patients with different treatment fractions were retrospectively investigated. All the patients were immobilized and localized with a stereotactic body frame and were treated with cone-beam CT guidance for each fraction. We calculated the actual delivered dose of the treatment plan using the up-to-date patient geometry of each fraction, and compared the dose with the intended plan dose to investigate the dosimetric effect of the inter-fraction deformation. Due to the relatively poor image quality of CBCT, deformable registration was carried out between treatment planning CT and CBCT of each fraction to obtain deformed planning CT for more accurate dose calculation of delivered dose. The extent of the inter-fraction deformation was also evaluated by calculating the dice similarity coefficient between the contours on planning CT and those on deformed planning CT.
\end{abstract}

Results: The average dice coefficients for PTV, spinal cord, esophagus were $0.87,0.83$ and 0.69 , respectively. The volume of PTV covered by prescription dose was decreased by $23.78 \%$ on average for all fractions of all patients. For spinal cord and esophagus, the volumes covered by the constraint

dose were increased by $4.57 \%$ and $3.83 \%$ in most fractions. The maximum dose was also increased by $4.11 \%$ for spinal cord and $4.29 \%$ for esophagus.

Conclusion: Due to inter-fraction deformation, large deterioration was found in both PTV coverage and OAR sparing, which demonstrated the need for adaptive re-planning of lung SBRT cases to improve target coverage while reducing radiation dose to nearby normal tissues.

Keywords: Lung SBRT; Inter-Fraction Deformation; Dosimetric Effect; Adaptive Re-Planning

TABLE 1: Evaluation metrics for OAR dose sparing.

\begin{tabular}{lccccc}
\hline \hline & Fx. \# 1 & Fx. \# 2 & Fx. \# 3 & Fx. \# 4 & Fx. \# 5 \\
\cline { 2 - 6 } PTV & 83.80 & 85.52 & 89.82 & 89.70 & 85.75 \\
\hdashline Spinal cord & 81.56 & 83.61 & 89.50 & 77.74 & 84.63 \\
\hdashline Esophagus & 59.66 & 70.09 & 82.83 & 63.61 & 66.62 \\
\hline \hline
\end{tabular}

Abbreviation: Fx. \# = Fraction Number

TABLE 2: Dice similarity coefficient comparison

\begin{tabular}{lll}
\hline \hline & 5 Fractions & 3 Fractions \\
\cline { 2 - 3 } Spinal cord & $\mathrm{V}_{4.6 G y}, \mathrm{~V}_{2.9 G y}, D_{\max }$ & $\mathrm{V}_{6 G \mathrm{G}}, \mathrm{V}_{4.16 y}, \mathrm{D}_{\max }$ \\
Esophagus & $\mathrm{V}_{3.9 G y}, \mathrm{D}_{\max }$ & $\mathrm{V}_{5.9 G y}, D_{\max }$ \\
\hline \hline
\end{tabular}

Presenting author: Jing Jia; Department of Radiation Oncology, UT Southwestern Medical Center, Dallas, TX, USA.

\section{Cite this article as:}

Jia J, Tian Z, Gu X, Yan H, Jia X, Jiang S. Investigating the dosimetric effect of inter-fraction deformation in lung cancer stereotactic body radiotherapy (SBRT). Int J Cancer Ther Oncol 2014; 2(2):020225. DOI: 10.14319/ijcto.0202.25
TABLE 3: PTV coverage

\begin{tabular}{lcccccc}
\hline \hline & Planning & Fx. \# & Fx. \# & Fx. \# & Fx. \# & Fx. \# \\
& CT & 1 & 2 & 3 & 4 & 5 \\
\cline { 2 - 7 } Volume $\left(\mathrm{cm}^{3}\right)$ & 44.56 & 37.36 & 42.52 & 47.31 & 43.46 & 42.24 \\
\cline { 2 - 7 } V100\%PD $(\%)$ & 83.98 & 78.59 & 76.95 & 76.71 & 77.77 & 76.28 \\
\hline \hline
\end{tabular}

Abbreviation: Fx. \# = Fraction Number 


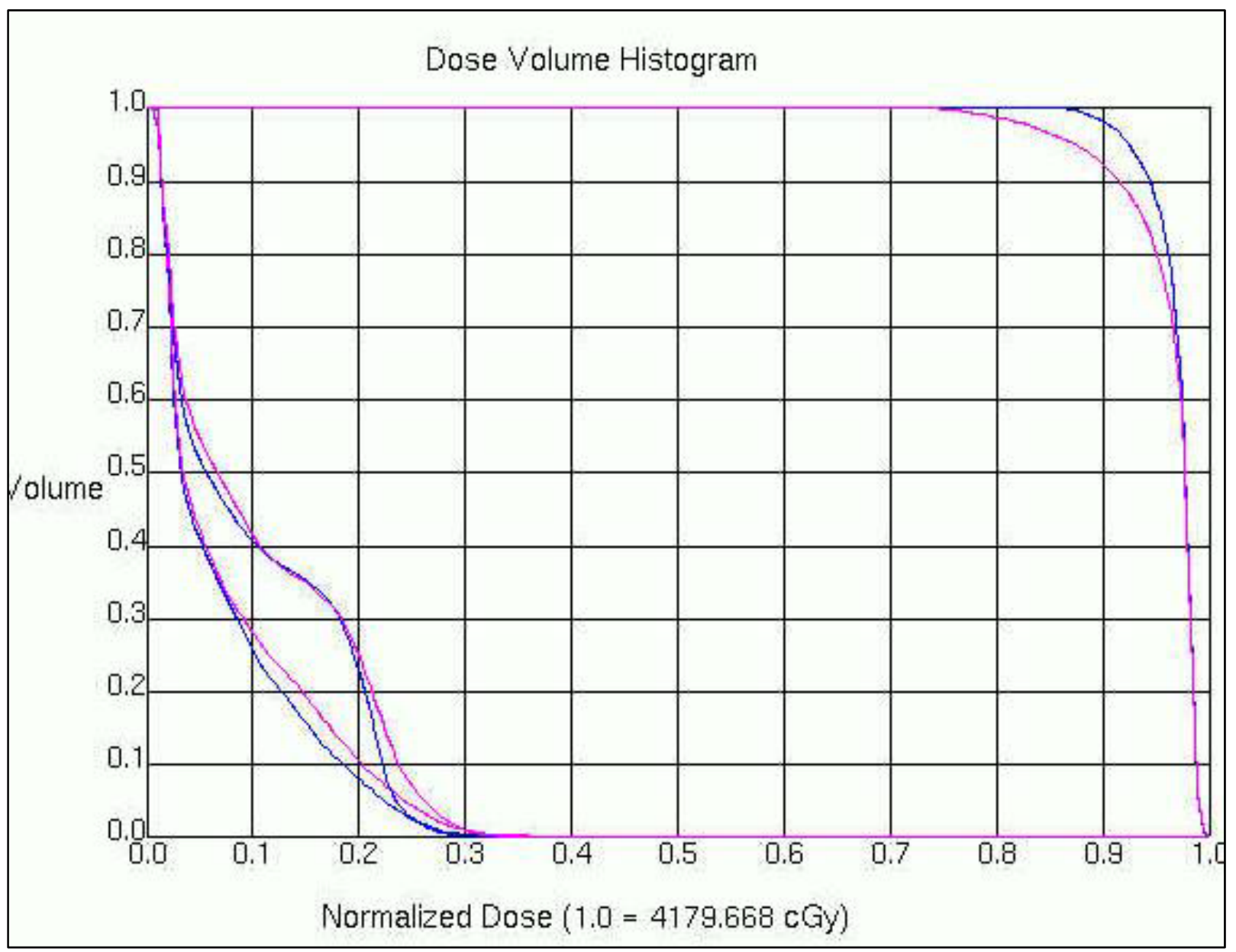

FIG. 1: DVH Comparisons for patient 1_Fraction 1 